\title{
A LIMIT ON THE SPACE DENSITY OF SHORT-PERIOD BINARY WHITE DWARFS
}

\author{
Edward L. Robinson and Allen W. Shafter \\ Department of Astronomy, University of Texas at Austin \\ Austin, Texas 78712
}

\begin{abstract}
We infer that detached binary white dwarfs with orbital periods of a few hours exist because we observe both their progenitors and their descendents. The binary LB 3459 has an orbital period of $6.3 \mathrm{hr}$ and contains a pair of hot subdwarfs that will eventually cool to become white dwarfs (Kilkenny, Hill, and Penfold 1981). L870-2 is a pair of white dwarfs and, given enough time, its $1.55 \mathrm{~d}$ orbital period will decay to shorter periods (Saffer, Liebert, and Olszewski 1988). GP Com, AM CVn, V803 Cen, and PG1346+082 are interacting binary white dwarfs with orbital periods between $1051 \mathrm{~s}$ for AM CVn and $46.5 \mathrm{~min}$ for GP Com (Nather, Robinson, and Stover 1981; Solheim et al. 1984; Wood et al. 1987; O'Donoghue and Kilkenny 1988). These ultrashort period systems must be descendents of detached pairs of white dwarfs. We also expect short-period binary white dwarfs to exist for theoretical reasons. All calculations of the evolution of binary stars show that main-sequence binaries can evolve to binary white dwarfs (e.g., Iben and Tutukov 1984). Among Population I stars, $1 / 2$ to $2 / 3$ of all main-sequence stars are binaries and about $20 \%$ of these binaries should become double white dwarfs with short orbital periods (Abt 1983, Iben and Tutukov 1986). Thus, about $1 / 10$ of all white dwarfs could be close binaries (Paczynski 1985). Nevertheless, no detached binary white dwarfs with extremely short periods have yet been found.

The space density of short-period binary white dwarfs is worth measuring for several reasons. Most generally, the space density is a test of modern theories for the evolution of binary stars. In addition, short-period binary white dwarfs should be the dominant source of gravitational radiation at periods between about $0.5 \mathrm{~min}$ and 1 hour (e.g., Clark and Epstein 1979, Evans, Iben and Smarr 1987). Plans to build detectors of gravitational radiation at these periods using space-based interferometers are now being seriously considered and this new generation of detectors should be sensitive enough to detect binary white dwarfs (Faller and Bender 1984). Finally, in the most commonly discussed model for Type Ia supernovae, the progenitor of the supernova is a short-period binary consisting of two white dwarfs with a total mass greater than the Chandrasekhar limit (Webbink 1979; Tutukov and Yungelson 1979; Iben and Tutukov 1984; Paczynski 1985; Tornambè and Matteucci 1986). Gravitational radiation drives the binary to shorter orbital periods and ultimately disrupts the lower mass white dwarf. If enough mass accretes onto the higher-mass white dwarf, its mass will exceed the Chandrasekhar limit and it will collapse
\end{abstract}


catastrophically. The space density of short-period binary white dwarfs is a direct test of this model.

We undertook, therefore, a search for binary white dwarfs among spectroscopically identified DA and DB white dwarfs (Robinson and Shafter 1988). As our primary goal was to test binary white dwarf models for Type Ia supernovae, we designed the survey to be sensitive to extremely short periods. Our basic method was to look for orbital radial velocity variations in the white dwarfs, although we chose an unusual instrumental technique for measuring the velocity variations. We used a highspeed photometer equipped with narrow band filters whose bandpasses lay in the wings of an absorption line, the H $\gamma$ line for DA white dwarfs or the He I $\lambda 4471$ line for DB white dwarfs. If the radial velocity of the white dwarf varies, the center of the absorption line moves toward or away from the filter bandpass, decreasing or increasing the flux of light through the filters. Orbital radial velocity variations can be detected from periodic variations in the filtered light curve. The amplitude of the flux variations can be converted to the amplitude of the radial velocity variation once the slope of the spectrum in the wing of the absorption line is known. Since all white dwarfs have surface gravities near $\log g=8$ and since the slopes do not depend strongly on the temperature of the white dwarf over the temperature range in which most white dwarfs are found, we used a typical value for the slopes to convert the flux variations to radial velocity variations. The slopes were taken from the theoretical line profiles calculated by Koester (1980) for the DB white dwarfs and by Wickramasinghe (1972) for the DA white dwarfs.

We observed 44 DA and DB white dwarfs chosen from the Villanova catalogue of spectroscopically identified white dwarfs (McCook and Sion 1987). The sample of stars consisted of white dwarfs that are brighter than $\mathrm{V}=14.5$, do not have close visual companions, and are observable from McDonald Observatory. There were no other selection criteria. We did not detect any binaries.

There are three significant limitations to our survey. First, because the temperatures of the narrow band filters were not actively controlled, their central wavelengths slowly drifted as the ambient temperature in the telescope dome changed, introducing systematic errors into the measured intensities. These errors became significant on time scales longer than three hours and, therefore, we limited our survey to orbital periods less than three hours. The short-period limit was $30 \mathrm{~s}$ and was set by the sampling interval in the flux measurements. Second, the fluxes through the narrow band filters were low, limiting our ability to detect small flux and radial velocity variations. Finally, if the two stars in a binary have similar brightness, our sensitivity to radial velocity variations decreases. If the spectral types of the two stars are different, the absorption line being measured is diluted by the continuum light from the other star and the slope of its wings and the flux variations are reduced. If the spectral types of the two stars are the same, the detected flux variations correspond to the weighted average of the radial velocity variations of the two stars. Since the radial 
velocities of the two stars have different signs, the averaging can reduce the flux variations and thus our sensitivity to radial velocity variations greatly.

It is impossible to know the exact effect of these various limits on our sensitivity to short-period binaries without knowing the distribution of orbital periods, spectral types, and masses of the binary white dwarfs, which is precisely what we do not know. The upper limits to the semi-amplitudes of the radial velocity variations are less than $70 \mathrm{~km} \mathrm{~s}^{-1}$ for 34 of the stars we observed and between 70 and $100 \mathrm{~km} \mathrm{~s}^{-1}$ for the remaining 10 stars. We have estimated the probability that we would have missed any binaries in our sample given these velocity limits by examining types of binaries that would be pathologically difficult to detect, generally binaries with periods at the longperiod limit containing white dwarfs of nearly equal luminosities. We find that we should have detected more than half of even the most difficult cases. Although there is a finite probability that we have missed some binaries in our sample, we believe that our sensitivity is good enough to have detected a large fraction, perhaps $90 \%$, of all binaries in our sample.

Our null result implies that the fraction of field white dwarfs that are binaries with periods less than 3 hours is less than $1 / 20$ with a probability of 0.9 . Since the properties of the white dwarfs in our sample are roughly similar to the properties of the white dwarfs in the sample used by Fleming, Liebert and Green (1986) to determine the space density and birth rates of white dwarfs, we can scale their results to obtain limits on the space density of binary white dwarfs. We find that the space density of binary white dwarfs with periods less than 3 hours and with $M_{V}<12.75$ is less than $3.0 \times 10^{-5} \mathrm{pc}^{-3}$ with a probability of 0.9 .

We test the binary white dwarf model for the progenitors of Type Ia supernovae by comparing the observed limit on the space density of binary white dwarfs to the space density required to produce the observed rate of Type Ia supernovae. If the observed rate of Type Ia supernovae per unit volume in the Galaxy is $R$ and if the orbital periods of the progenitor binary white dwarfs decay at a rate $d P / d t$, the distribution of binary white dwarfs in period is

$$
n(P)=R\left(\frac{d P}{d t}\right)^{-1} .
$$

We assume that the only mechanism causing the orbits to decay is gravitational radiation and that most of the binary white dwarfs first emerge from their common envelope evolution at periods longer than 3 hours. The required space density of binary white dwarfs with orbital periods less than 3 hours is given by the integral of $n(P)$ over orbital periods between 0 and 3 hours.

The rate of Type Ia supernovae in the galaxy is not well known. Using the historical supernovae, Tammann (1982) derives a rate of $0.027 \mathrm{yr}^{-1}$ for the entire Galaxy. Using a subset of the historical supernovae, van den Berg (1983) and van den Berg, McClure, and Evans (1987) derive a rate of $0.022 \mathrm{yr}^{-1}$ for all supernovae. 
Somewhat over half of this rate is due to Type I supernovae, from which we can derive a rate of roughly $0.012 \mathrm{yr}^{-1}$ for Type I supernovae alone. Note that the observed ratio of Type I to Type II supernovae in the Galaxy is not the same as the ratio in other galaxies, almost certainly because many Type II supernovae in the Galaxy have been missed. We convert the total rate to a volume rate by the usual method. The effective area of the Galactic disk is $10^{9} \mathrm{pc}^{3}$ (Miller and Scalo 1979). According to Tammann (1982) the observed scale height of Type I supernovae about the Galactic disk is about $200 \mathrm{pc}$. Alternatively, if we assume that Type Ia supernovae belong to an extremely old disk population, a scale height as large as $400 \mathrm{pc}$ may be more appropriate. The value of $\mathrm{R}$ could, then, reasonably lie anywhere between $1.5 \times 10^{-14}$ and $6.8 \times 10^{-14} \mathrm{yr}^{-1}$ $\mathrm{pc}^{-3}$. The latter value is the one given by Tammann (1982). We adopt $R=(4.0 \pm 2.5) \mathrm{x}$ $10^{-14} \mathrm{yr}^{-1} \mathrm{pc}^{-3}$.

We this choice of $R$, the required space density of binary white dwarfs that will become Type Ia supernovae is $(1.6 \pm 1.0) \times 10^{-5} \mathrm{pc}^{-3}$. On theoretical grounds we expect that only about $1 / 10$ of all binary white dwarfs are pairs of Carbon-Oxygen white dwarfs with total masses high enough to become Type Ia supernovae (Iben and Tutukov 1986) and, therefore, the required space density of progenitors for Type Ia supernovae must be multiplied by 10 to give the a required space density of all shortperiod binary white dwarfs. The number that is to be compared to our observed limit is, then, $(1.6 \pm 1.0) \times 10^{-4} \mathrm{pc}^{-3}$. The required space density is a factor of $5 \pm 3$ greater than our observed limit.

Our result is evidence that there is something amiss in the simplest binary white dwarf models for the progenitors of Type Ia supernovae. There are several possibilities for what could be wrong; among them:

1) The measured rate of Type Ia supernovae in the Galaxy may be too high. The rate is based on a small number of historical supernovae in the galaxy, 7 in Tammann's sample and 3 in van den Berg's sample, and requires uncertain corrections for incompleteness. A large error in the rate would, nevertheless be surprising because with reasonable choices for the luminosity of the Galaxy and for the Hubble constant the galactic rate now agrees with the rate for external galaxies (van den Berg, McClure, and Evans 1987).

2) Binary white dwarfs may be only one of several different progenitors for Type Ia supernovae. Iben and Tutukov (1984) have catalogued the possible alternative progenitors. One alternative, interacting helium star-white dwarf pairs, is particularly attractive as these systems could have a high enough birth rate to account for Type Ia supernovae if a large fraction of these systems become Type Ia instead of Type Ib supernovae (Iben and Tutukov 1987; Iben, Nomoto, Tornambè, and Tutukov 1987).

3) We have assumed that binary white dwarfs all form at orbital periods longer than 3 hours. Theoretical calculations (e.g., Webbink 1984) suggest that some fraction of the binaries should form at periods less than 3 hours. Because binaries formed at shorter periods have shorter lifetimes, the space density of these short period 
systems would be low. The fraction of systems that are formed at periods less than 3 hours is uncertain because the physics of common-envelope evolution is uncertain, but a fraction larger than one-half may require a major revision in our understanding of common envelope evolution.

4) If the binaries have special properties, they could have been missed because of the way we selected the stars to observe. A magnitude limited survey like ours is biased towards intrinsically bright stars. If most of the progenitors of Type Ia supernovae have absolute visual magnitudes fainter than about 13.0 they will be under-represented in our sample. Also, if the progenitors are pairs of DC white dwarfs, they would not be included in our sample.

5) Finally, some technical problems could arise in comparing the space density of binary white dwarfs to the birth rate for Type Ia supernovae. If any mechanism for removing orbital angular momentum from the system besides gravitational radiation is operating, the orbits will decay faster and the required space density of binary white dwarfs will be reduced. No reasonable alternative to gravitational radiation has been suggested, however. We have adopted a value of $1 / 10$ for the ratio of the number of binary white dwarfs with total mass large enough to make supernovae to the number with mass too small to make supernovae. It would be necessary to invoke a peculiar IMF at early times to change the ratio by a large factor. If the distribution of binary white dwarfs in orbital periods is not in a steady state, the relation between $n(P)$ and $R$ that we have adopted is not correct. This could happen if the star formation rate in the galaxy (or at least in the neighborhood of the sun) has been far from uniform.

This research was supported in part by NSF Grant AST-8704382.

\section{References}

Abt, H. A. 1983, Ann. Rev. Astr. Ap., 21, 343.

Clark, J.P.A., and Epstein, R. 1979, in Sources of Gravitational Radiation, ed. L. L. Smarr (Cambridge: Cambridge University Press), p. 482.

Evans, C. R., Iben, I., and Smarr, L. 1987, Ap.J., 323, 129.

Faller, J. E., and Bender, P. L. 1984, in Precision Measurement and Fundamental

Constants II, ed. by B. N. Taylor and W. D. Phillips (NBS Spec. Pub. 617).

Fleming, T. A., Liebert, J., and Green, R. F. 1986, Ap. J., 308, 176.

Iben, I., Nomoto, K., Tornambè, A., and Tutukov, A. V. 1987, Ap. J., 317, 717.

Iben, I., and Tutukov, A. V. 1984, Ap. J. Suppl., 54, 335.

Iben, I., and Tutukov, A. V. 1986, Ap. J., 311, 753.

Iben, I., and Tutukov, A. V. 1987, Ap. J., 313, 727.

Kilkenny, D., Hill, P. W., and Penfold, J. E. 1981, M.N.R.A.S., 194, 429.

Koester, D. 1980, Astr. Ap. Suppl. , 39, 401.

McCook, G. P., and Sion, E. M. 1987, Ap. J. Suppl., 65, 603.

Miller, G. E., and Scalo, J. H. 1979, Ap. J. Suppl., 41, 513.

Nather, R. E., Robinson, E. L., and Stover, R. J. 1981, Ap. J., 244, 269. 
O'Donoghue, D., and Kilkenny, D. 1988, M.N.R.A.S., in press.

Paczynski, B. 1985, in Cataclysmic Variables and Low-Mass X-Ray Binaries, ed. D. Q. Lamb and J. Patterson (Dordrecht: Reidel), p. 1.

Robinson, E. L., and Shafter, A. W. 1987, Ap. J., 322, 296.

Saffer, R. A., Liebert, J., and Olszewski, E. W. 1988, Ap. J., in press.

Solheim, J. E., Robinson, E. L., Nather, R. E., and Kepler, S. O. 1984, Astr. Ap., 135, 1.

Tammann, G. A. 1982, in Supernovae: A Survey of Current Research, ed. M. J. Rees and R. J. Stoneham (Dordrecht: Reidel), p. 371.

Tournambè, A., and Matteucci, F. 1986, M.N.R.A.S., 223, 69.

Tutukov, A. V., and Yungelson, L. R. 1979, Acta Astr., 29, 665.

van den Berg, S. 1983, P.A.S.P., 95, 388.

van den Berg, S., McClure, R. D., and Evans, R. 1987, Ap. J., 323, 44.

Webbink, R. F. 1979, in IAU Colloquium No. 53, White Dwarfs and Variable Degenerate Stars, ed. H. M. Van Horn and V. Weidemann (Rochester: University of Rochester), p. 426.

Webbink, R. F. 1984, Ap.J., 277, 355.

Wickramasinghe, D. T. 1972, Mem. R. A. S., 76, 129.

Wood, M. A., Winget, D. E., Nather, R. E., Hessman, F. V., Liebert, J. W., Kurtz, D. W., and Wesemael, F. 1987, Ap.J., 313, 757. 